\title{
STRUCTURAL EFFECTS ON SYNCHRONIZABILITY OF SCALE-FREE NETWORKS
}

\author{
JIAN-GUO LIU* and TAO ZHOU ${ }^{\dagger}$ \\ Department of Modern Physics and Nonlinear Science Center \\ University of Science and Technology of China \\ Hefei 230026, P. R. China \\ Department of Physics, University of Fribourg \\ Chemin du Musée 3, CH-1700 Fribourg, Switzerland \\ *liujg004@ustc.edu.cn \\ †zhutou@ustc.edu \\ QIANG GUO \\ Dalian Nationalities University \\ Dalian 116600, P. R. China \\ guoqiang@dlnu.edu.cn \\ BING-HONG WANG \\ Department of Modern Physics and Nonlinear Science Center \\ University of Science and Technology of China \\ Hefei 230026, P. R. China \\ bhwang@ustc.edu.cn
}

\begin{abstract}
In this paper, we numerically investigate the structural characteristics that affect the synchronizability of coupled identical oscillators on scale-free networks. By using the edge-exchange method, we can change the network structure with degree sequence fixed. An optimal algorithm, namely Tabu Search, is applied, respectively, to enhance and weaken the synchronizability. The numerical results indicate that the synchronizability is most sensitive to the maximal betweenness. By tracking the optimization process, most previous works suggest that the synchonizability can be enhanced by reducing assortative coefficient and clustering coefficient; however, interestingly, our numerical simulations show that the depression of synchronizability also corresponds to the reduction process of those two coefficients.
\end{abstract}

Keywords: Scale-free networks; synchronizability; assortative coefficient.

PACS Nos.: 89.75.Da, 05.45.-, 05.45.Xt. 


\section{Introduction}

Synchronization in coupled oscillators, extensively observed in a variety of natural, social, physical, and biological systems, has been studied for many years. ${ }^{1-8}$ Recent empirical studies have demonstrated that many real-world networks are small-world ${ }^{9}$ and scale-free, ${ }^{10}$ thus cannot be treated purely as regular or random networks. As network structure has significant impacts on the dynamical processes, an increasing effort has been devoted to the exploration of synchronization phenomena in complex networks. ${ }^{11-14}$

Previous works indicated that homogeneous network has stronger synchronizability, ${ }^{15-17}$ but the strict and clear conclusions have not been achieved. On the basis of an ideal mathematical model, Zhou et al. ${ }^{18}$ found that the average distance, $D$, is a key factor to the synchronizability. The maximal betweenness, $B_{\max }$, has also been extensively studied. Some numerical results suggested that the network with smaller $B_{\max }$ would have stronger synchronizability; ${ }^{16,17,19}$ however, a counterexample is reported recently ${ }^{20}$ against this oversimple conclusion. In addition, Zhou et al. ${ }^{21}$ studied the phase synchronization of community networks and found that the community structure will hinder global synchronization. Actually, a network contains countless topological measures, such as degree distribution $P(k)$, average distance $D$, clustering coefficient $C$, maximal betweenness $B_{\max }$, and so on. In most of the previous works, if one wants to show the effect of a structural characteristic on synchronizability, he/she would tune this characteristic and monitor the change of synchronizability while keeping others approximately fixed. ${ }^{22}$ Using the edge-exchange operation, presented by Maslov et al. ${ }^{23}$ Donetti et al. ${ }^{24}$ optimized the network synchronizability from different initial configurations and found that the optimization process will lead to the same optimal result, namely Entangle Network. In this paper, combine the Tabu Search (TS) algorithm ${ }^{25}$ and the edge-exchange method, ${ }^{20,23,26,27}$ we enhance and weaken the synchronizability of scale-free networks with degree sequence fixed. The numerical results indicate that the synchronizability of scale-free networks is most sensitive to $B_{\max }$. In addition, different from most previous works, which suggest that the synchonizability can be enhanced by reducing assortative coefficient ${ }^{20,28}$ and clustering coefficient, ${ }^{20,27,29}$ our numerical simulations show that the depression of synchronizability also corresponds to a reduction process of those two coefficients.

\section{Synchronizability}

In this section, we will introduce a generic model of coupled oscillators on networks and a useful measure ${ }^{30}$ to quantify the stability of the global synchronized states. Consider a network of $N$ linearly coupled identical oscillators, the dynamics reads

$$
\dot{\mathbf{x}}^{i}=\mathbf{F}\left(\mathbf{x}^{i}\right)-\sigma \sum_{j=1}^{N} G_{i j} \mathbf{H}\left(\mathbf{x}^{j}\right), \quad i=1, \ldots, N
$$


where $\dot{\mathbf{x}}^{i}=\mathbf{F}\left(\mathbf{x}^{i}\right)$ governs the local dynamics of the $i$ th oscillator, $\mathbf{H}\left(\mathbf{x}^{i}\right)$ is the output linear vectorial function, $\sigma$ is the coupling strength, and $G=\left\{g_{i j}\right\}$ is the Laplacian matrix, where

$$
g_{i j}= \begin{cases}k_{i}, & i=j, \\ -1, & i \in \Lambda_{i}, \\ 0, & \text { otherwise. }\end{cases}
$$

Here $\Lambda_{i}$ is the set of all $i$ s neighbors. Because the symmetry and the positive semidefinite of $G$, all its eigenvalues are non-negative real values and the smallest eigenvalue $\lambda_{0}$ is always zero. The eigenvalues can be ranked as $0=\lambda_{0}<\lambda_{1} \leq \lambda_{2} \leq \cdots \leq \lambda_{N}$.

Stability of the synchronous state $\mathbf{x}^{i}(t)=\mathbf{x}^{s}(t)(i=1, \ldots, N)$ can be accounted for by diagonalizing the linear stability equation, yielding $N$ blocks of the form $\dot{\zeta}_{i}=\left[\mathbf{J F}\left(\mathbf{x}_{s}\right)-\sigma \lambda_{i} \mathbf{H}\left(\mathbf{x}_{s}\right)\right] \zeta_{i}$, where $\mathbf{J}$ is the Jacobian operator, $\zeta_{i}$ is the eigenmode associated with the eigenvalue $\lambda_{i}$ of $G$ and $i=1,2, \ldots, N$. Replacing $\sigma \lambda_{i}$ by $\nu$ in the equation, the master stability function $(\mathrm{MSF})^{30}$ fully accounts for the linear stability of the synchronization manifold. For a large class of oscillatory systems, the MSF is negative in a finite parameter interval $I_{s t} \equiv\left(\nu_{1} \leq \nu \leq \nu_{2}\right) .{ }^{30}$ When the whole set of eigenvalues (multiplied by $\sigma$ ) enters the interval $I_{s t}$, the stability condition is satisfied. This is accomplished when $\sigma \lambda_{2}>\nu_{1}$ and $\sigma \lambda_{N}<\nu_{2}$ simultaneously. As $\nu_{2}$ and $\nu_{1}$ depend on the specific choice of $\mathbf{F}(\mathbf{x})$ and $\mathbf{H}(\mathbf{x})$, the key quantity for assessing the synchronizability of a network is the eigenratio

$$
Q=\frac{\lambda_{N}}{\lambda_{2}},
$$

which relies on the topology only. The smaller the $Q$, the easier to synchronize the oscillators, and vice versa. ${ }^{31}$

According to the MSF, ${ }^{30}$ the eigenratio $Q$ can measure the network synchronizability, when the stable zone is bounded. When the stable zone is unbounded, synchronizability could be measured by the maximal nonzero eigenvalue $\lambda_{\max } \cdot{ }^{13}$

In this paper, we will not address a particular dynamical system but concentrate on how the network topology affects the eigenratio $Q$, with an assumption that the stable zone is bounded.

\section{Algorithm and Numerical Results}

The procedures of the edge-exchange operation in an undirected network are given as follows:

- Randomly pick two existing edges $e_{1}=\left(v_{1}, v_{2}\right)$ and $e_{2}=\left(v_{3}, v_{4}\right)$. The four nodes $\left\{v_{1}, v_{2}, v_{3}, v_{4}\right\}$ must be different, and there is no edge connecting $v_{1}$ and $v_{4}$, as well as $v_{2}$ and $v_{3}$.

- Exchange these two edges to obtain $e_{1}^{\prime}=\left(v_{1}, v_{4}\right)$ and $e_{2}^{\prime}=\left(v_{2}, v_{3}\right)$.

We take the eigenratio $Q$ as the objection to minimize or maximize, and study the relationship between the synchronizability and structural characteristics during the 
optimization process via a TS algorithm. Due to the use of the tabu list to keep several standard optimal objects, TS algorithm is much more efficient than the classical Monte Carlo (MC) algorithm. From the viewpoint of statistical mechanics, TS and MC algorithms are very similar. The processes of TS algorithm, weakening the eigenratio $Q$, is demonstrated as follows (the enhancing process is similar):

Step 1. Generate a BA network ${ }^{10}$ with $N$ nodes and $E$ edges, and denote its coupling matrix by $G_{0}$. Set the optimal coupling matrix $G_{k}^{*}=G_{0}$, the optimal network of tabu table $G_{k}=G_{0}$, and the time step $k=0$. Compute $Q$ of $G_{k}^{*}$.

Step 2. Randomly chose two edges and intercross them, denote the new coupling matrix by $G$.

Step 3. If $Q_{G} \leq Q_{G_{k}^{*}}, G_{k+1}^{*}=G, G_{k+1}=G$, else if $Q_{G} \leq Q_{G_{k}}, G_{k+1}=G$, else if $G$ does not satisfy the tabu condition $\left|Q_{G_{k}}-Q_{G}\right| / Q_{G}>\delta$ (where $\delta$ is a random number between 0.5 and 0.75$)$, then $G_{k+1}=G$, else $G_{k+1}=G_{k}$. Go to Step 2.

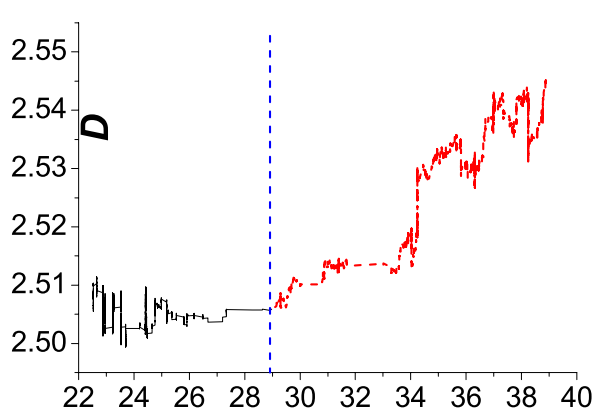

(a)

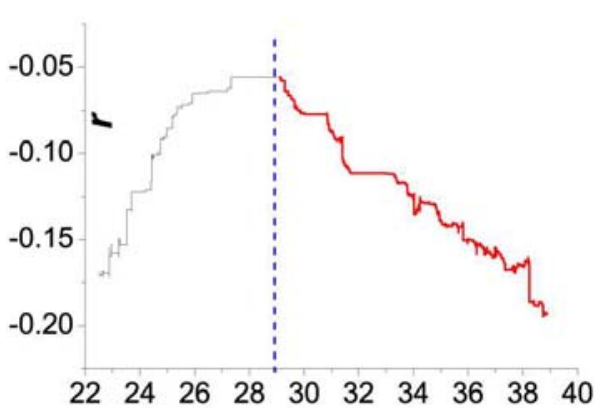

(c)

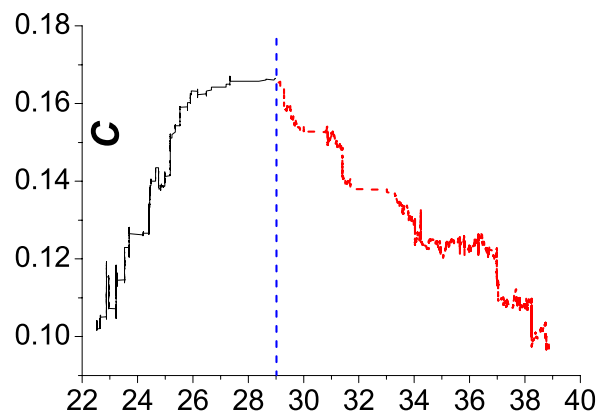

(b)

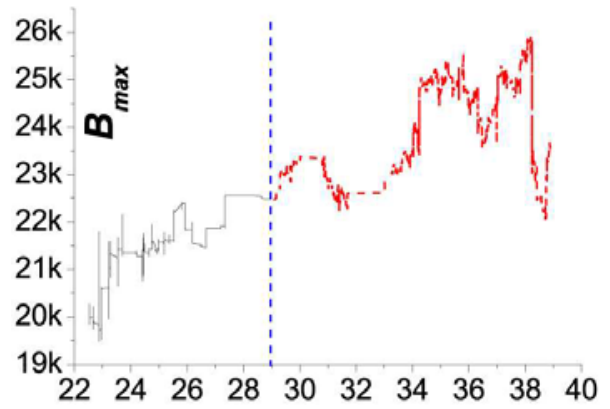

(d)

Fig. 1. (Color online) The structural characteristics versus the eigenratio $Q$. (a) average distance $D$; (b) clustering coefficient $C$; (c) assortative coefficient $r$; and (d) maximal node betweeness $B_{\max }$. The black and red curves represent the processes on enhancing and depressing synchronizability, respectively. Blue dashed line denotes the initial condition. 
Start from $N=500$ and the average degree $\langle k\rangle=6$. At each time step, we record the structural properties, including average distance $D$, clustering coefficient $C$, assortative coefficient $r$, and maximal node betweenness $B_{\max }$. Figure 1 gives the changing process of the structural measures when enhancing and weakening the eigenratio $Q$. Figure 1(a) demonstrates that $D$ remains stable when weakening $Q$, whereas it increases a little when enhancing $Q$. Figures $1(\mathrm{~b})$ and $1(\mathrm{c})$ show the changing procedure of $C$ and $r$, from which one can find that in both the enhancing and weakening processes, $C$ and $r$ decrease from the initial value. Figure 1(d) shows the change procedure of $B_{\max }$. When weakening $Q, B_{\max }$ decreases, on the contrary, $B_{\max }$ gets larger value than the initial value and fluctuate greatly. With the increase in $Q$, this result indicates that the network with higher synchronizability would have smaller $B_{\max }$.

It should be emphasized that the edge-exchange method could solely suppress the assortative coefficient. Therefore, the decrease in the assortative coefficient presented in Fig. 1 may not be aroused by the change of the network synchronizability. Figure 2 demonstrated the change process of assortative coefficient, $r$, solely aroused by the edge-exchange method. From which one can find that $r$ would decrease to about -0.22 at 5000 time steps. However, the numerical simulation results presented in Fig. 1 shows that $r$ would not decrease beyond -0.2 . Although the difference is very small, we cannot get the conclusion that the assortative coefficient $r$ is a key factor to network synchronizability.

\section{Conclusion and Discussions}

In summary, using the TS algorithm, we maximized and minimized the network synchronizability by changing the connection pattern between different pairs of nodes while keeping the degree sequence unchanged. This work is based on the MSF with a fundamental assumption that the essential dynamics can be locally linearized. The eigenratio $Q$ is used to measure the network synchronizability. Besides the MSF, some other methods, such as the time series analysis, ${ }^{32,33}$ are proposed to evaluate the synchronizability. Though its own limitation, the MSF is, thus far, widely accepted as a standard analytical tool for network synchronization. Especially, it avoids the details embedded in the idiographic dynamics. Anyway, it is worthwhile to warn the readers that all the analyses presented here are only valid under the framework of MSF.

The numerical results indicate that $D, C, r$, and $B_{\max }$ influence network synchronizability simultaneously. Especially, the network synchronizability is most sensitive to $B_{\max }$. By tracking the optimization process, most previous works suggest that the synchonizability can be enhanced by reducing assortative coefficient ${ }^{20,28}$ and clustering coefficient. ${ }^{20,29,27}$ However, our numerical simulations show that the depression of synchronizability also corresponds to a reduction process of clustering coefficient. It should be emphasized that the decrease in the assortative coefficient may be caused by the edge-exchange method. Although there is a small difference 
between the deceasing ranges caused by the synchronizability optimal process and the edge-exchange, respectively, we cannot get a conclusion that the assortative coefficient would decrease enhancing or weakening the network synchronizability. The significant and interesting result [see Fig. 1(b)] gives rise to a challenge about the mentioned claims. Although a completely clear picture about the roles of structural characteristics on synchronizability is not obtained here, the present idea about the comparison between the enhancing and weakening processes may be useful to distinguish/judge the validity of prior conclusions.

By using the optimal algorithm, we can expect to reveal the common structural characteristics of networks with nearly maximal synchronizability. For example, Donetti et al. ${ }^{24}$ found that the more homogeneous the distributions of degree and node distance, the stronger the synchronizability, and Wang et al. ${ }^{27}$ concluded that the networks of stronger synchronizability should have lower clustering coefficient and fewer low-order loops. In addition, we can track the optimization process to detect the possible trends of structural change. One should note that, since all the structural characteristics change simultaneously, mathematically speaking, it is impossible to obtain the strict conclusions (e.g., recently, by using the classical graph theory, Duan et al. ${ }^{34}$ presented us some counterexamples to the widely accepted conclusions). However, the observed changing trends are statistically reasonable for the large-scale real-world networks (as shown in Fig. 2(d) about the changing of $\left.B_{\max }\right)$. Actually, there are countless topological measures, and no one can keep all others unchanged while tuning one monitored measure, unless the analytical tools can be as accurate as microscope - knowing the exact change of any measures induced by adding one node or rewiring one edge. However, it is not feasible when

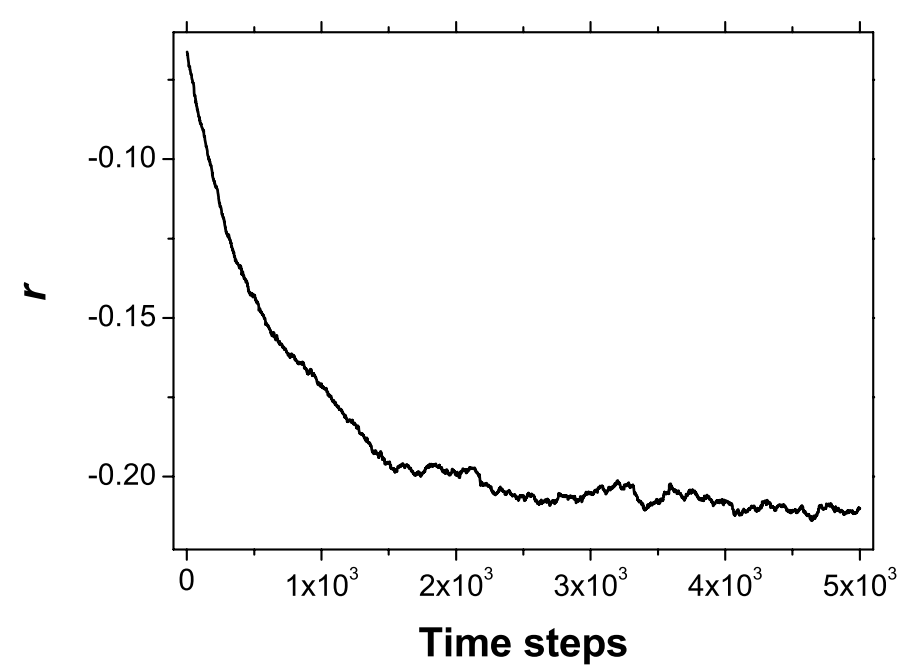

Fig. 2. $\quad r$ versus the randomly edge-exchange process without any dynamical functions. All the data are obtained by 10 independent runs. 
analyzing the real networks with thousands to millions nodes. Therefore, we believe that this work could shed some light to the readers in this interesting field.

\section{Acknowledgments}

The authors thank Wenxu Wang, Guanrong Chen, and Bing Wang for their valuable comments and warm discussions. This work is funded by the National Basic Research Program of China (973 Program No. 2006CB705500), the National Natural Science Foundation of China (Grant Nos. 10635040, 10532060, 60744003 and 10472116), the Theoretical Physics Frontier Problems (NSFC Grant No. A0524701), and the Specialized Research Fund for the Doctoral Program of Higher Education of China.

\section{References}

1. S. Strogatz, SYNC-How the Emerges from Chaos in the Universe, Nature, and Daily Life (Hyperion, New York, 2003).

2. L. M. Pecora and T. L. Carroll, Phys. Rev. Lett. 64, 821 (1990).

3. K. M. Cuomo and A. V. Oppenheim, Phys. Rev. Lett. 71, 65 (1993).

4. H. G. Winful and L. Rahman, Phys. Rev. Lett. 65, 1575 (1990).

5. K. Otsuka, R. Kawai, S. L. Hwong, J. Y. Ko and J. L. Chern, Phys. Rev. Lett. 84, 3049 (2000).

6. D. Hansel and H. Sompolinsky, Phys. Rev. Lett. 68, 718 (1992).

7. M. de S. Vieira, Phys. Rev. Lett. 82, 201 (1999).

8. J. Hemmingsson, A. Sorensen, H. Flyvbjerg and H. Herrmann, Eusophys. Lett. 23, 629 (1993).

9. D. J. Watts and S. H. Strogatz, Nature 393, 440 (1998).

10. A.-L. Barabási and R. Albert, Science 286, 509 (1999).

11. L. F. Lago-Fernández, R. Huerta, F. Corbacho and J. A. Siguenza, Phys. Rev. Lett. 84, 2758 (2000).

12. P. M. Gade and C.-K. Hu, Phys. Rev. E 62, 6409 (2000).

13. X. F. Wang and G. Chen, Int. J. Bifurcation Chaos Appl. Sci. Eng. 12, 187 (2002).

14. P. G. Lind, J. A. C. Gallas and H. J. Herrmann, Phys. Rev. E 70, 056207 (2004).

15. T. Nishikawa, A. E. Motter, Y.-C. Lai and F. C. Hoppensteadt, Phys. Rev. Lett. 91, $014101(2003)$.

16. H. Hong, B. J. Kim, M. Y. Choi and H. Park, Phys. Rev. E 69, 067105 (2004).

17. M. Zhao, T. Zhou, B.-H. Wang and W.-X. Wang, Phys. Rev. E 72, 057102 (2005).

18. T. Zhou, M. Zhao and B.-H. Wang, Phys. Rev. E 73, 037101 (2006).

19. S. Jalan and R. E. Amritkar, Phys. Rev. Lett. 90, 014101 (2003).

20. M. Zhao, T. Zhou, B.-H. Wang, G. Yan, H.-J. Yang and W.-J. Bai, Physica A 371, $773(2006)$.

21. T. Zhou, M. Zhao, G. Chen, G. Yan and B.-H. Wang, Phys. Lett. A 368, 431 (2007).

22. M. Zhao, T. Zhou, B.-H. Wang, Q. Ou and J. Ren, Eur. Phys. J. B 53, 375 (2006).

23. S. Maslov and K. Sneppen, Science 296, 910 (2002).

24. L. Donetti, P. I. Hurtado and M. A. Muñoz, Phys. Rev. Lett. 95, 188701 (2005).

25. F. Glover, Computers and Operation Research 5, 533 (1986).

26. B. J. Kim, Phys. Rev. E 69, 045101 (2004).

27. B. Wang, T. Zhou, Z. L. Xiu and B.-J. Kim, Eur. Phy. J. B 60, 89 (2007). 
28. M. di Bernardo, F. Carofalo and F. Sorrentino, Int. J. Bifurcation Chaos Appl. Sci. Eng. 17, 2419 (2007).

29. X. Wu, B.-H. Wang, T. Zhou, W.-X. Wang, M. Zhao and H.-J. Yang, Chin. Phys. Lett. 23, 1046 (2006).

30. L. M. Pecora and T. L. Carroll, Phys. Rev. Lett. 80, 2109 (1998).

31. M. Barahona and L. M. Pecora, Phys. Rev. Lett. 89, 054101 (2002).

32. A. Kabakciog̈lu, I. Kanter, M. Vendruscolo and E. Domany, Phys, Rev. E 65, 041903 (2001).

33. R. Q. Quiroga, T. Kreuz and P. Grassberger, Phys. Rev. E 66, 041904 (2002).

34. Z. Duan, G. Chen and L. Huang, Phys. Rev. E 76, 056103 (2007). 